\title{
ACQUE, CAMPI E BOSCHI NELLA CIVILTÀ DELLE TERRAMARE. LE RAGIONI DI UN COLLASSO DI CIVILTÀ NELLA PIANURA PADANA DELL'ETÀ DEL BRONZO
}

\author{
MAURO CREMASCHI (*)
}

\begin{abstract}
SuNTO. - La civiltà delle Terramare si sviluppò al centro della valle padana nell'età del Bronzo medio e recente (1550-1150 anni a.C.). Si trattò di una cultura complessa ed articolata che mise in opera una strategia di sfruttamento territoriale audace ed innovativa, introducendo per la prima volta in pianura padana l'agricoltura intensiva irrigua. In un paesaggio radicalmente deforestato, per rispondere alla necessità di aver ampi spazi da coltivare e per poter disporre di grandi quantità di legname per costruire i villaggi, la gestione delle acque ebbe un ruolo di primo piano. A questo fine venne realizzata una fitta rete di canali e fossi irrigui che provvedeva all'irrigazione della campagna circostante i villaggi. La civiltà delle terramare scompare in breve tempo nel Bronzo recente avanzato; ci si interroga ancora sulle ragioni di questa crisi che ha probabilmente le sue radici nello sfruttamento delle risorse oltre ai limiti della sostenibilità, ma che precipita forse per la concausa di un breve, ma drammatico periodo di siccità.
\end{abstract}

$* * *$

ABSTRACT. - The Terramare civilization spread in the middle of the Po plain during the middle and recent Bronze Ages (1550-1150 BC). It resulted in a complex and articulate culture that promoted a innovative territorial exploitation strategy, introducing for the first time in the Po plain the intensive agriculture based on irrigation. The area of Terramare was radically deforested to respond to the need for ample space of cultivated land and to provide timbers for the construction of the villages. Water management played a prominent role: a dense net of ditches and canals provided to irrigate the land surrounding the settlements. The civilization of Terramare collapsed at the end of the recent Bronze age The reasons for this crisis are still questioned, they probably have their roots in exploiting resources beyond the limits of sustainability. However a short but dramatic arid spell acted as decisive cause to unleash the collapse.

(*) Dipartimento di Scienze della Terra dell’Università di Milano, via Mangiagalli 34, Milano, Italia. E-mail: mauro.cremaschi@unimi.it 


\section{INTRODUZIONE}

Le terramare sono i resti archeologici di villaggi che risalgono alla media e alla recente età del Bronzo (1550-1150 a.C.) (Bernabò Brea, Cardarelli, 1997a) (Fig. 1).

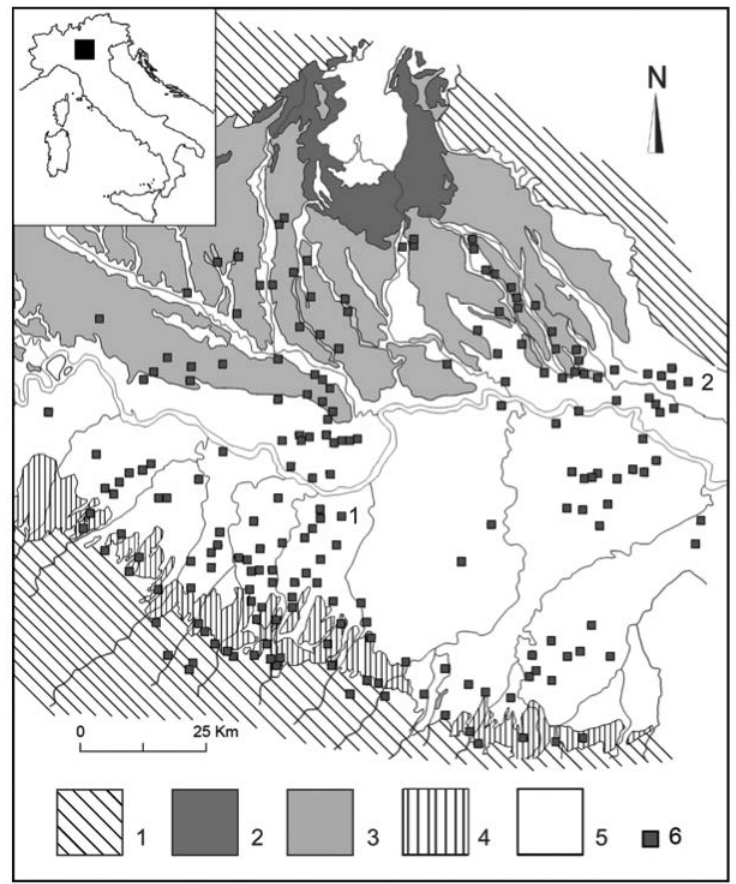

Fig. 1. Distribuzione delle terramare e siti coevi nella pianura padana centrale.

1 - aree collinari dell' Appennino e delle Prealpi lombarde e venete; 2 - depositi morenici di età glaciale del lago di Garda; 3 - livello fondamentale della pianura lombarda (depositi fluvioglaciali); 4 - terrazzi pleistocenici del margine appenninico; 5 - pianura olocenica; terramare e siti coevi: 1. Santa Rosa di Poviglio; 2. Fabbrica dei Soci.

Il nome deriva dal fatto che vennero scoperte perché utilizzate come cava di terra fertile a partire dal XVIII e per gran parte del XIX secolo (Bernabò Brea, Mutti, a cura di, 1994, Pearce, 1998). Tali siti archeologici sono testimonianze di una civiltà articolata e complessa (vedasi Bernabò Brea; Cardarelli; Cremaschi; a cura di, 1997, e bibliografia citata), protagonisti della prima stagione dell'archeologia preistorica 
italiana, sono oggi di nuovo al centro di numerose iniziative di scavi, studi e ricerche che ne approfondiscono sostanzialmente le conoscenze circa la cultura materiale, la complessità degli abitati, l'ampiezza degli scambi, il contesto ambientale. Un tema centrale sul quale si concentra l'attenzione degli studiosi è l'improvvisa crisi con la quale questa civiltà si conclude, attorno al 1150 a.C, per cause ad esse interne, come l'incremento demografico, l'uso non sostenibile delle risorse, ma anche probabilmente per un concomitante sfavorevole cambiamento climatico. Oggi sappiamo che le terramare hanno svolto un ruolo di primo piano nel quadro della preistoria europea perchè hanno introdotto, per la prima volta in Europa, un sistema di gestione territoriale che prevedeva l'agricoltura cerealicola intensiva sorretta dall'irrigazione.

\section{TERRAMARE, BOSCHI E CAMPI}

Confrontando i diagrammi pollinici di siti terramaricoli nella valle padana a quelli di altri coevi (Fig. 2), si osserva che gran parte dell'Italia, nel momento di massima fioritura delle terramare è coperta da una densa coltre forestale e solo l'area terramaricola (l'Emilia, e parte della Lombardia, del Veneto e della Romagna) risulta radicalmente deforestata.

L'elevata domanda di legno che giustifichi una così intensa deforestazione è innanzitutto determinata dal numero e dalle dimensioni (alcuni superavano i venti ettari di estensione) dei villaggi che erano prevalentemente costruiti con questo materiale. Di legno erano costruite le case all'interno degli abitati (su impalcato nelle prime fasi, appoggiate al suolo in fase tarda), le recinzioni, le infrastrutture (recinzioni, palizzate, ponti). Da calcoli, pure sommari, condotti sulla terramara di Santa Rosa, risulta che il solo impianto di una terramara di medie dimensioni (senza pensare alle successive manutenzioni e cambiamenti di planimetria) richiedeva l'abbattimento di ettari di bosco (Cremaschi, 2009).

Le poche fotografie che mostrano le strutture lignee conservate nelle terramare esplorate nel XIX secolo scorso (Castellazzo di Fontanellato, Castione dei Marchese: vedasi per il materiale iconografico: Bernabò Brea, Cardarelli, Cremaschi, a cura di, 1997) e soprattutto la recente fortunata scoperta della vasca votiva di Noceto (Bernabò Brea, Cremaschi, 2009) danno un'idea concreta dell'importanza del 
legno come materiale da costruzione, della perizia degli artigiani e del largo uso di questo materiale in molteplici forme.

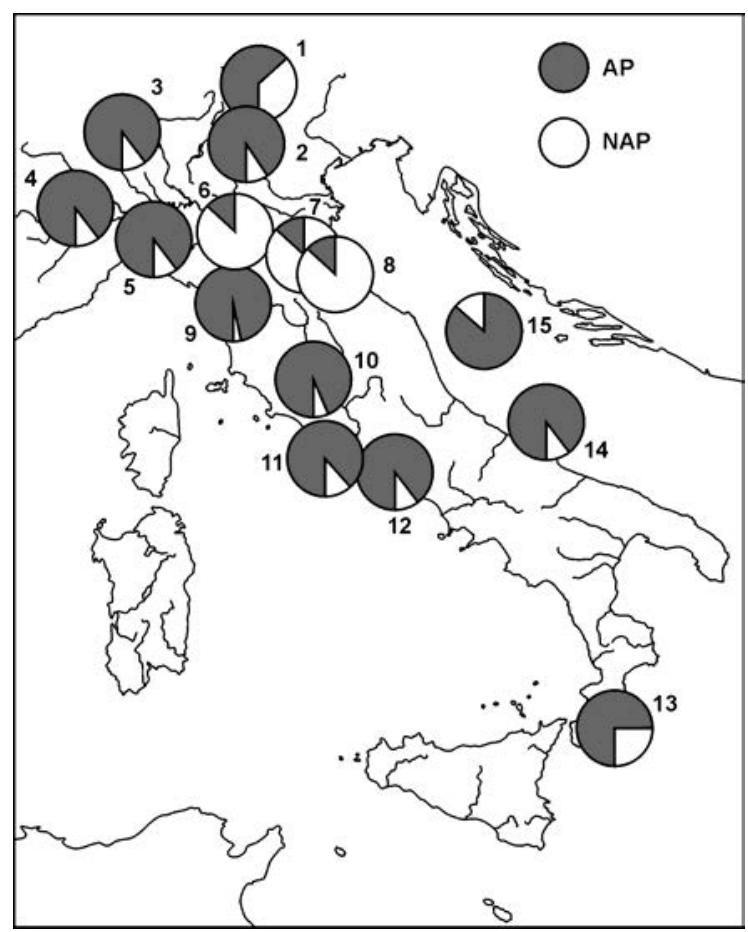

Fig. 2. I boschi in Italia durante la media e recente età del Bronzo. I diagrammi circolari rappresentano in grigio l'incidenza delle piante arboree (AP) ed in bianco l'incidenza delle erbacee, ivi compresi i cereali (NAP) (da Cremaschi et al. 2006). 1 - Fiavè; 2 - Lago del Frassino; 3 - Biandronno e Ganna; 4 - Prato Mollo; 5 - Prato Spilla;

6 - Terramara Santa Rosa di Poviglio; 7 - Terramara di Montale; 8 - Monte Castellaccio; 9 - Lago Padule; 10 - Lagaccione e Lago Vico; 11 - Lago Albano;

12 - Valle di Castiglione; 13 - Canolo Nuovo; 14 - Adriatico centrale, carotaggio pal 94 -8; 15 - Adriatico centrale, carotaggio pal 9330.

La domanda di legname per costruire gli abitati non costituisce la sola causa dell'intensa deforestazione che è anche legata alla domanda di spazi aperti da dedicare all'agricoltura. L'attività di deforestazione, non è esclusiva dell'età del Bronzo, inizia in pianura padana con la tecnica dello slash and burn durante il Neolitico, limitatamente a piccoli appezzamenti; prosegue nell'età del Rame con grandi 
incendi verosimilmente estesi su ampie aree (Cremaschi, Nicosia, 2012). Le conseguenze sul manto vegetale di queste deforestazioni non sono durature e la copertura forestale si rigenera tempestivamente, dominando nei diagrammi pollinici di questo periodo. Solo con la civiltà delle terramare si consolida l'esigenza di terreni stabilmente coltivati e la deforestazione diventa permanente.

Tra il Bronzo medio ed il Bronzo recente l'insediamento terramaricolo va incontro ad un'ampia riorganizzazione (Bernabò Brea, Cardarelli, 1997) che porta all'aumento del numero complessivo dei siti, ma soprattutto al notevole ampliamento di alcuni di loro, come ad esempio la terramara di Santa Rosa, che passa da uno a sette ettari di estensione per effetto dell'aumentata pressione demografica. L'ampiezza delle aree deforestate cresce di conseguenza. Attorno alla terramara (Ravazzi et al. 2004) durante il Bronzo medio, il territorio deforestato si estendeva per un raggio di almeno cinquecento metri, mentre nell'età del Bronzo recente avrebbe raggiunto un raggio di almeno quattro chilometri.

Estrapolando la situazione di Santa Rosa da altri siti terramaricoli della bassa valle dell'Enza, in cui il paesaggio dell'età del Bronzo è particolarmente ben conservato, è possibile osservare che durante il Bronzo medio potevano ancora sussistere fra sito e sito ampie aree coperte di bosco, mentre queste si ridussero severamente o scomparvero del tutto nel Bronzo recente, limitando di fatto la disponibilità di nuovi territori nei quali estendere la coltivazione. Inoltre, a fronte di un enorme consumo, la disponibilità di legname si è avvicinata ad una soglia critica nelle fasi più avanzate della cultura terramaricola. Non è certamente un caso che negli strati superiori della terramara di Santa Rosa (Bernabò Brea, Cremaschi, 2004; Bianchi, 2004) che datano al Bronzo recente avanzato, le case non fossero più poste su impalcato, ma appoggiate a terra e quindi costruite con una tecnica meno esigente in fatto di legname, indicandone una diminuita disponibilità.

Lo stabilirsi dei gruppi terramaricoli nella pianura padana avviene attraverso una sorta di 'colonizzazione' di un area prima scarsamente insediata e dà luogo da subito ad una fitta rete di insediamenti: si rende quindi necessario provvedere, mediante pratiche di agricoltura intensiva, al mantenimento della fertilità dei suoli a garanzia dei raccolti.

A questo fine i terramaricoli introducono l'agricoltura irrigua, poiché l'irrigazione, oltre a fornire l'acqua necessaria al germogliare delle sementi, arricchisce il suolo di molteplici nutrienti, specialmente organici (Mercuri et al. 2006 b). 
In alcuni recenti interventi di Archeologia Preventiva, nell'area del conoide dell'Enza sono venute in luce, lontano dagli insediamenti maggiori, tratti di canali irrigui e pozzi per acqua isolati, che attestano un paesaggio intensamente coltivato. Cortile S. Martino, presso Parma, ne resta comunque l'esempio più significativo (Cremaschi 2009): in questa località è venuto in luce (Bernabò Brea et al. 2007) un suolo debolmente sviluppato, sepolto nella pianura alluvionale a un metro circa di profondità, con tracce di deforestazione mediante incendio, associato a piccoli edifici risalenti alla media età del Bronzo. Su questo suolo ed in concomitanza ai resti archeologici, vi è una rete di canalette che, incrociandosi, appaiono delimitare dei campi e risultano alimentate da pozzi che si approfondiscono anche per tre metri fino ad intercettare la falda idrica.

L'uso dell'aratro nelle terramare è ampiamente attestato (Forni, 1997) anche grazie agli esemplari rinvenuti nella vasca di Noceto (Bernabò Brea, Cremaschi, 2009). Questo strumento, grazie all'utilizzo della forza animale, amplifica largamente la velocità di lavorazione del suolo rispetto alla vanga e alla zappa in uso nelle più antiche culture (Forni, 1997) ed è quindi ben probabile che, in associazione all'agricoltura irrigua, abbia costituito un motore tecnologico di significativo progresso, che ha permesso agli agricoltori terramaricoli di produrre ie surplus alimentare da investire nelle numerose altre attività svolte all'interno del gruppo sociale, che vanno dallo scavo dei canali alla costruzione degli insediamenti strutturati alle attività artigianali specializzate (metallurgia, ceramica, oggetti di pregio, ecc...).

I suoli non venivano (Ravazzi et al. 2004; Mercuri et al. 2006 b) concimati mediante stallatico, ma il ripristino dei nutrienti era affidato alla pratica della rotazione.

Nella serie pollinica recentemente studiata nel fossato del Villaggio Piccolo della terramara di Santa Rosa (Cremaschi et al. 2016) si susseguono livelli nei quali i picchi polliniei di cereali si alternano a picchi di piante nitrofile che testimoniano appunto i momenti in cui i campi non erano coltivati, ma lasciati a pascolo e frequentati da caprovini.

Della superficie disboscata (Ravazzi et al. 2004; Mercuri et al. 2006a) solo il trenta o il quaranta per cento veniva destinato all'agricoltura ed il resto costituiva i prati pascoli destinati a sostenere la fauna domestica, largamente dominante nello spettro zooarcheologico dei siti terramaricoli. Le specie principalmente allevate sono caprovini, bovini 
e suini. E' significativo che nel Bronzo recente (sia a Santa Rosa che al Montale, anche se in un momento leggermente precedente), aumentino, a scapito dei suini, i caprovini (e tra questi le capre sulle pecore) che prediligono spazi aperti e possono tollerare pascoli più siccitosi (De Grossi Mazzorin, Riedel, 1997; Riedel, 2004).

\section{TERRAMARE, FOSSATI E CORSI D’ACQUA}

Una stretta relazione fra terramare e corsi d'acqua era già stata osservata dai ricercatori del XIX secolo. Nell'idea iniziale di Chierici (1871) gli abitati nelle vicinanze di un corso d'acqua avrebbero derivato mediante un canale l'acqua per alimentare il bacino artificiale delimitato da un terrapieno, all'interno del quale sarebbe stato collocato il villaggio oppure, come si affermò più tardi, il canale avrebbe dovuto alimentare un fossato, ma in posizione esterna rispetto al terrapieno. In entrambi i casi, a valle dell'abitato, un altro canale avrebbe provveduto a far riconfluire l'acqua nel corso originario.

Le ricerche, condotte a partire degli anni settanta dello scorso secolo, hanno pienamente confermato questo modello (Cremaschi, 1997).

Sulle foto aeree di molte località della pianura padana si disegnano evidenti, come nel celebre esempio di Fabbrica dei Soci (Fig. 3) (Balista, 1997, 2002), i perimetri quadrangolari dei terrapieni degli abitati maggiori, circondati da un ampio fossato, connesso alle tracce paleoidrografiche di antichi corsi d'acqua. Anche in assenza di queste, i fossati sono comunque alimentati o dal drenaggio minore, ad esempio i corsi da risorgive, come nel caso della terramara di Beneceto (Bernabò Brea et al. 2004) o direttamente dai fontanili, come nel caso della terramara di Case del Lago (Cremaschi, 1997).

L'abitato difeso da terrapieno e circondato da un fossato aveva, per le terramare, certamente lo scopo di sottolinearne la monumentalità dell'insediamento nel paesaggio e di rispondere a necessità di difesa (Cremaschi, Pizzi 2013, Cremaschi et al. 2016 b) ma, per quanto riguarda i fossati, questo non era certamente il solo scopo per il quale vennero costruiti. Infatti dal fossato della terramara di Castello del Tartaro (Balista 1997) una rete di piccoli canali artificiali esce radialmente dal fossato verso la campagna con l'evidente scopo di regolare la distribuzione idraulica verso i campi circostanti (irrigare in tempi di siccità, drenare nei casi di eccesso idrico). Il fenomeno ricorre siste- 
maticamente per tutte le terramare in cui vi sia stata la possibilità di esplorarne le periferie (Case del Lago, Vicofertile, Case Cocconi, ma anche Gaggio e Santa Rosa di Poviglio) (Balista 1997; Balista, De Guio 1997; Balista 2002; Balista et al. 2003; Cremaschi 1997). I grandi villaggi terramaricoli appaiono avere un ruolo di governo dei territori ad essi circostanti gestendone l'acqua, la più preziosa risorsa per un paesaggio rurale indirizzato principalmente alla coltura cerealicola.

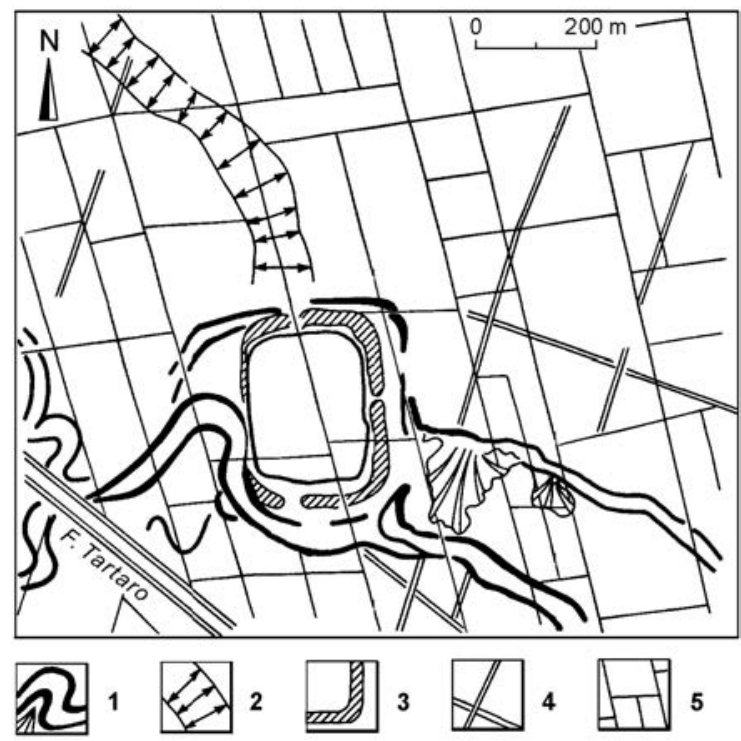

Fig. 3. Immagine fotoaerea della terramara Fabbrica dei Soci (Valli Grandi veronesi). 1 - Paleoalveo del Tartaro; 2 - dosso fluviale; 3 -terrapieno della terramara ed antistante fossato; 4 - tracce di centuriazione romana; 5 - parcellazione agraria moderna.

Gli apparati idraulici venuti in luce al margine meridionale del Villaggio Grande della terramara di Santa Rosa (Cremaschi et al. 2006; Pizzi, Cremaschi 2004; Cremaschi, Pizzi 2007) (Fig. 4), offrono una prova archeologica agli esempi citati, basati soprattutto su evidenze geomorfologiche. Il Villaggio Grande della terramara di Poviglio, al suo apogeo (Bronzo recente), ha una complessa organizzazione: l'area dell'abitato, costituita da case rettangolari su impalcato (Fig. 5), è delimitata da una palizzata posta al margine del fossato che circondava l'intero sito. Tra le case, sono state identificate due 
strade che escono dal villaggio, attraversando due porte, testimoniate dalle tracce di struttura lignea rimaste impresse nel terreno (Bernabò Brea et al. 2003).

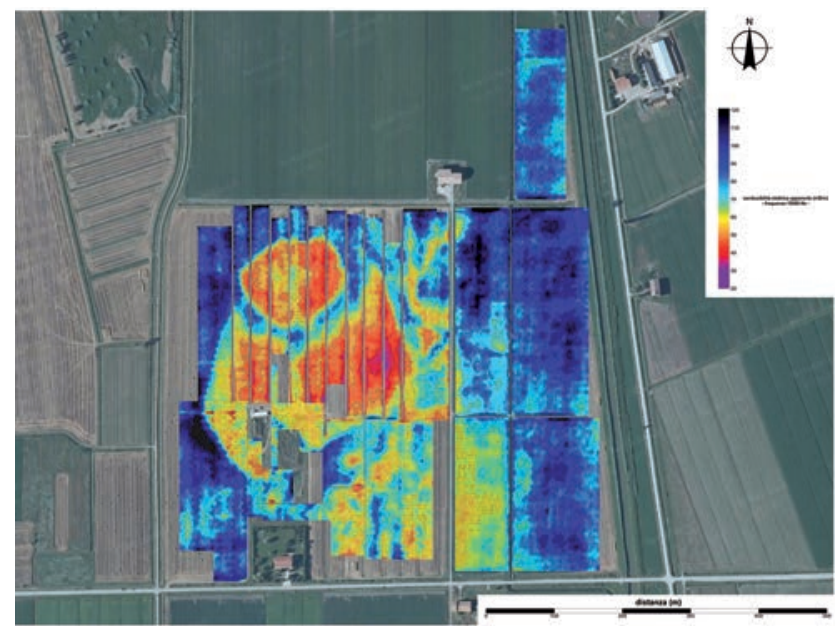

Fig. 4. Immagine del rilievo geofisico della Terramara di Santa Rosa.

I colori rossi ed arancione (terreni conduttivi) indicano l'area della terramara, distinguendo chiaramente il Villaggio Piccolo a Nord ed il Villaggio Grande a Sud.

I colori blu indicano le aree resistive principalmente costituite da terreni argillosi che colmano i cavi circostanti la terramara. Si notino in particolare i fossati che circondano sia il Villaggio Piccolo che il Villaggio Grande (da Mele et al., 2013).

Lungo la palizzata sono venuti in luce numerosi grandi pozzi che attingevano acqua da un acquifero posto a -3 metri dalla superficie topografica attuale. I pozzi sono rimasti in uso a lungo (dal Tardo Bronzo medio fino al Bronzo recente in base al contenuto archeologico) (Cremaschi, Pizzi 2007) e riescavati numerose volte. L'acqua però non veniva condotta all'interno del villaggio stesso ma, attraverso un complesso sistemi di piccoli canali, riversata nel fossato.

Il fossato che si trova all'esterno della recinzione è ampio, poco profondo ed asimmetrico, ripido ed inaccessibile dalla parte del villaggio, ma dolcemente inclinato verso la campagna. Durante il Bronzo recente, le acque provenienti dai pozzi della recinzione venivano raccolte alla base del fossato e fatte scorrere verso Sud Est. In questo modo il fossato, di norma approvvigionato da acque di falda, aveva maggiori 
probabilità di disporne anche durante la stagione secca. Nel fossato tuttavia convergeva anche un ampio canale proveniente da Sud Ovest. Questo è largo quattro metri, profondo quasi due metri e le sue pareti sono inclinate e gradonate per aumentarne la stabilità. Con tutta probabilità questa struttura corrisponde all' incile' previsto dal modello ottocentesco delle terramare ed era destinata a portare acqua nel fossato da un vicino corso. Le strade che escono dall'abitato attraversano sia il fossato che il canale su strutture di passaggio, identificabili con ponti che grazie alla particolare struttura agivano anche da regolatori del flusso idrico che scorreva nei due cavi (Pizzi, Cremaschi, 2004; Cremaschi, Pizzi 2007, 2013). L'analisi geomorfologica suggerisce che il canale adduttore potesse prendere origine da un paleoalveo dell'Enza che correva ad almeno due chilometri dalla terramara. Si sarebbe trattato perciò di un'opera di grande impegno, come del resto le altre opere infrastrutturali, e suggerisce che i terramaricoli disponessero di un'articolata struttura organizzativa per progettare l'opera e reperire le risorse per realizzarla.

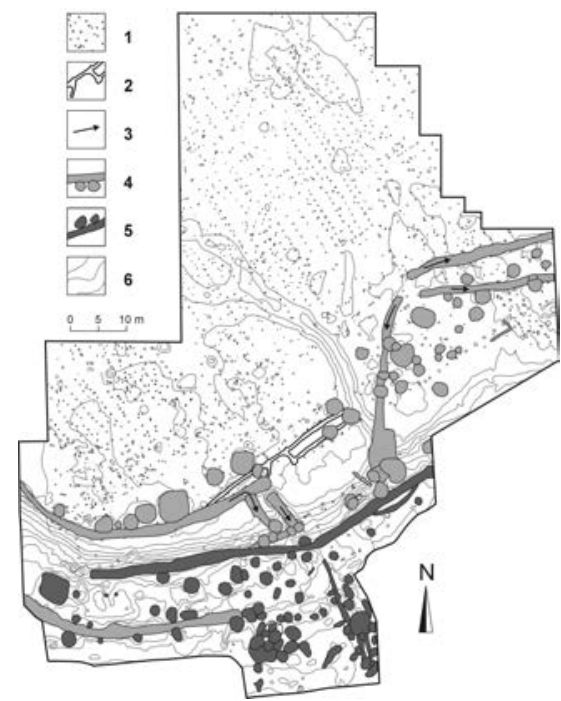

Fig. 5. Terramara di Santa Rosa, area del Villaggio Grande ed antistante fossato. 1 - buche di palo dell'abitato del Villaggio Grande, si riconoscono le planimetrie degli edifici su impalcato; 2 - tracce delle strutture lignee pertinenti alle porte; 3 - direzioni di drenaggio; 4 - pozzi della recinzione attivi tra l'età del Bronzo medio avanzato ed il Bronzo recente; 5 - Pozzi e strutture all'interno del fossato attivi nel Bronzo recente avanzato; 6 - isobate definenti la forma del fossato. 
Tra i molti cambiamenti che avvengono al margine del Villaggio Grande nella sua fase finale (Bronzo recente avanzato) c'è la defunzionalizzazione dei pozzi della recinzione ed il loro seppellimento al di sotto del terrapieno che va a sostituire la recinzione lignea (Pizzi, Cremaschi 2004; Cremaschi, Pizzi 2007). I pozzi del margine non sono più in grado di alimentare il fossato a causa dell'abbassamento della falda idrica. Di conseguenza sul fondo del fossato stesso, ora prosciugato, viene aperto un gran numero di nuovi pozzi nel tentativo di attingere acqua da una nuova falda, più profonda di almeno due metri rispetto ai pozzi della recinzione (Fig. 6).

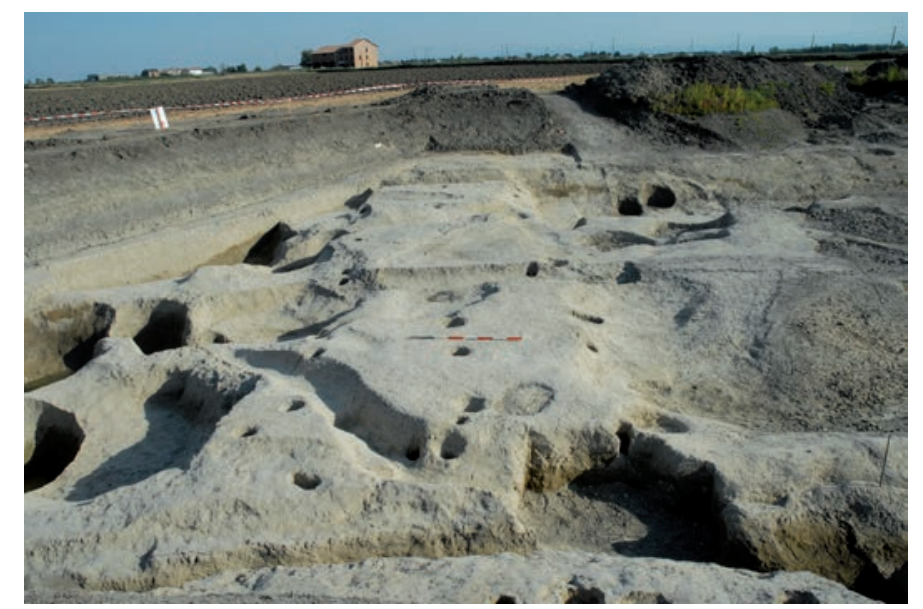

Fig. 6. Terramara Santa Rosa di Poviglio. 'Ponte'orientale attraversante il fossato del Villaggio Grande. Si noti sulla sinistra la fitta rete di grandi pozzi che datano alla fine del

Bronzo recente e cercano di attingere alla falda idrica per far fronte all'aridità.

I pozzi sul fondo del fossato sono collegati fra loro da canalette atte a ridistribuirne l'acqua e sono inframmezzati da cisterne poco profonde, spesso quadrangolari, che in un caso conservano ancora le buche di palo che potrebbero aver costituito le basi per apparati di estrazione dell'acqua (del tipo degli shadouf nordafricani), e si raccolgono in gruppi addossati specialmente al margine esterno del fossato stesso, in modo da servire specialmente la campagna esterna al villaggio, ad esempio per farvi scendere il bestiame da abbeverare.

I pozzi del fossato indicano l'abbassamento della falda idrica, e pertanto un periodo di aridità che potrebbe aver avuto un rilievo 
almeno regionale poiché le falde della terramara sono controllate dal Po che è il principale collettore idraulico del bacino padano. In verità, sulla base di recenti ricerche paleoclimatiche, un marcato episodio di aridità è registrato in questo momento (circa 1150 anni a.C.) anche da archivi paleoclimatici più generali, come ad esempio l'andamento del radiocarbonio residuale e gli speleotemi di cavità dell'Italia settentrionale (Cremaschi et al. 2006; Valsecchi et al. 2008; Magny 2004, Holzhauser et al. 2005, Regattieri et al. 2014).

\section{CONCLUSIONI: UNA CRISI DALLE MOLTEPLICI CAUSE}

La coincidenza fra periodo arido e l'abbandono di gran parte degli abitati terramaricoli, ivi compresa la terramara Santa Rosa di Poviglio, spinge a cercarvi un nesso causativo e a proporre una interpretazione in chiave climatica della dibattuta questione del collasso delle terramare (De Marinis 1975; Bernabò Brea et al. 1997b; Pearce 1998; Cremaschi et al. 2006, Cremaschi 2009 b) (Fig. 7).

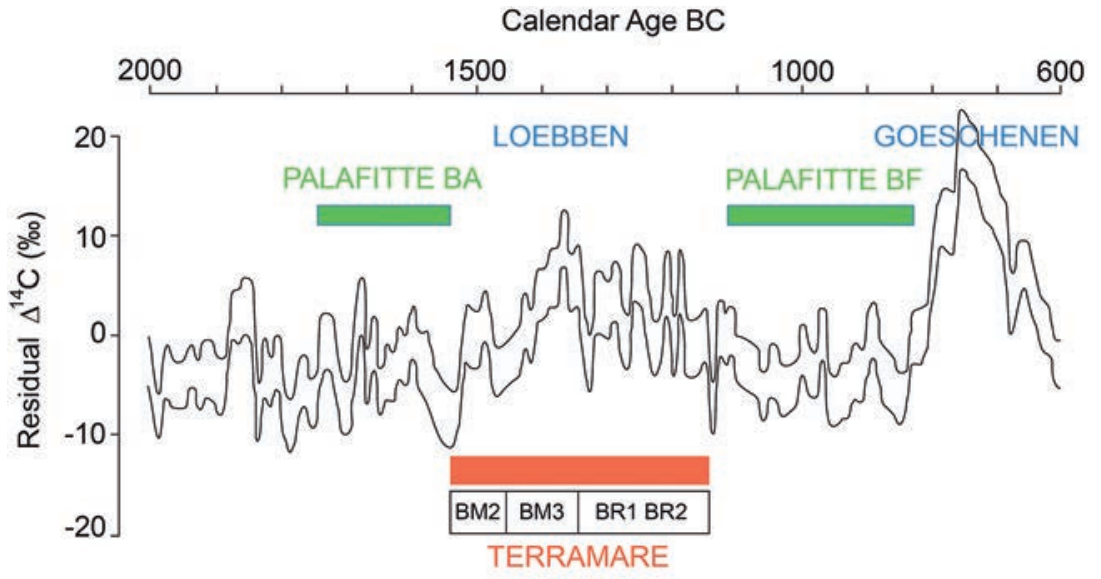

Fig. 7. Cambiamenti climatici del tardo Olocene, come suggerti dalle variazioni del Carbonio 14 residuale. Sono indicati in verde i periodi delle palafitte alpine (svizzere, tedesche, francesi) del Bronzo antico (BA) e finale (BF) ed i periodi di sviluppo delle terramare. Sono indicati i periodi di avanzata dei gbiacciai alpini, Loebben e Goeschenen che si sovrappongono alle vicende dell'età del Bronzo alpine e padane. Si noti il breve ma pronunciato picco negativo di 14 C residuale, indicante un evento arido, che coincide con la fine delle Terramare. 
Tuttavia è improbabile che una breve crisi arida, sia pure intensa, (Orombelli 1997; Magny 2004) possa aver causato da sola il crollo di una civiltà complessa assestata da centinaia di anni. Allora, come già sostenuto da alcuni autori (Pearce 1998), bisogna rivolgersi alle dinamiche economiche e sociali della cultura terramaricola e, specialmente, alle sue strategie di sfruttamento delle risorse ambientali. Con tutta probabilità l'evento climatico ha agito da catalizzatore facendo precipitare una situazione già compromessa (Cremaschi et al. 2006). Infatti, a ben vedere, numerosi indizi di crisi nello sfruttamento delle risorse si affacciano durante il Bronzo recente. Nella palafitta di Parma ci si rivolge in fase tarda al miglio (Panicum miliaceum), coltura meno esigente del tritticum, largamente usato in precedenza, (Lancelotti 2004/2005). Un peggioramento nel rendimento agricolo è indiziato al Montale (Mercuri et al. 2006 b) ed a Santa Rosa (Cremaschi et al., 2016 a) dove, nelle fasi più tarde, la coltivazione di cereali declina sensibilmente, mentre si amplia l'estensione dei campi non coltivati. Inoltre l'aumento nelle faune dei capriovini a discapito di bovini e suini, documentata nell'ultima fase delle terramare, indica anch'esso una maggiore aridità ma anche una estensione degli incolti.

E'perciò possibile che alcuni anni di accentuata aridità, magari in concomitanza con fattori d'ordine economico e sociale, abbiano contribuito a precipitare una situazione già spintasi ben oltre le soglie della sostenibilità, in un territorio fortemente impoverito ed al tempo stesso gravato da una forte pressione demografica.

Del resto non mancano esempi storicamente documentati di effetti negativi sulle comunità umane indotti da eventi aridi. Tra i tanti, mi pare particolarmente significativo ricordare il quadro che Le Roy Ladurie (2004) traccia per la Francia del XIII secolo, in occasione del ripetersi di estati molto calde, con il loro concatenarsi di eventi sfavorevoli: raccolti scarsi, acqua scarsa ed infetta per il bestiame e gli umani ed il conseguente scatenarsi di pestilenze. Riferendosi all'esempio di Le Roy Ladurie, un evento pestilenziale drammatico potrebbe effettivamente essere evocato ( $\mathrm{ma}$ è difficile reperirne prove concrete) per le terramare e giustificarne il collasso così repentino ed il prolungato tabù che avrebbe impedito di rioccupare, per ben quattro secoli, il territorio nel quale erano fiorite. 


\section{BIBLIOGRAFIA}

BALISTA, C., 1997. Fossati, canali e paleoalvei: connessioni nevralgiche per l'impianto e la sopravvivenza dei grandi siti terramaricoli di bassa pianura. In: Bernabò Brea, M., Cardarelli, A., Cremaschi, M. a cura di, Le terramare. La più antica civiltà padana. Electa, Milano, 126-136.

BALISTA, C., 2002. La paleoidrografia dell'area terramaricola centro-padana verso la fine dell'età del Bronzo: inquadramento stratigrafico, cronologico e paleoclimatico. Quaderni della bassa modenese 42, 15, 2, 7-48.

Balista C., De Guio A., 1997. Ambienti ed insediamento dell'età del Bronzo nelle Valli Grandi Veronesi, in Bernabò Brea M., Cardarelli A., Cremaschi M., a cura di, 1997, Le Terramare. La più antica civiltà padana, Milano, Electa, 137-170.

Balista C., Cardarelli A., Steffè G., Labate D., Mazzoni C., 2003. Terramara di Gaggio - Castelfranco Emilia (MO), in Bernabò Brea M., Valloni R., a cura di, Archeologia ad Alta Velocità in Emilia Romagna. Indagini archeologiche e geologiche lungo il tracciato ferroviario ad Alta Velocità, Atti della giornata di studi, Parma, 120-155.

Bernabò Brea M., MutTi A., a cura di, 1994, “... Le terramare si scavano per concimare i prati...”. La nascita dell'archeologia preistorica a Parma nella seconda metà dell'Ottocento, catalogo della mostra, Parma, Artegrafica Silva, 245-251.

Bernabò Brea M., Cardarelli A., Cremaschi M., a cura di, 1997. Le Terramare. La più antica civiltà padana, Milano, Electa.

BeRnabò BrEa M., CARDARELli A., 1997 a. Le terramare nel tempo, in Bernabò Brea M., Cardarelli A., Cremaschi M. a cura di 1997, Le Terramare. La più antica civiltà padana, Milano, Electa., 295-379.

Bernabò BREA M., CARDARELli A., CREMASCHI M., 1997 b. Il crollo del sistema terramaricolo, Bernabò Brea M., Cardarelli A., Cremaschi M., a cura di, 1997, Le Terramare. La più antica civiltà padana, Milano, Electa, 745-753.

Bernabò Brea M., Cremaschi M., Pizzi C., 2003. Le strutture abitative del Villaggio Grande-fase su palafitta- della terramara di S. Rosa di Poviglio (RE), in Atti del Convegno "Analisi informatizzata e trattamento dati delle strutture di abitato di età Preistorica e Protostorica in Italia”, 271-285.

Bernabò Brea M., Cremaschi M., a cura di, 2004. Il Villaggio Piccolo della Terramara Santa Rosa a Poviglio. Scavi 1987-1992, Origines, Firenze.

Bernabò Brea M., Cremaschi M., Bronzoni L., Pavia F., Rovesta C., 2007. Soil use from Late Calcolithic to the Early Middle Bronze Age. New data from buried soils of the middle Po Plain (Northern Italy), in Hidden landscapes of Mediterranean Europe. Cultural and methodological biases in pre-and protobistoric landscape studies, Atti del Convegno, Siena, 123-135.

Bernabò Brea M., Cremaschi M., a cura di, 2009. Acque e Civiltà nelle terramare. La Vasca votiva di Noceto. Università degli Studi di Milano, Le finestre del Sapere 7, Skira, Milano.

BIAnCHI P., 2004. Capanne e spazi domestici del Bronzo Recente avanzato nel Villaggio grande della terramara di S. Rosa di Poviglio, Rivista di Scienze Preistoriche LIV, pp. 411-485.

CHIERICI G., 1871. Le antichità preromane della provincia di Reggio nell'Emilia, Reggio nell'Emilia, Tip. di S. Calderini. 
CREmaschi M., 1997. Terramare e paesaggio padano, Bernabò Brea M., Cardarelli A., Cremaschi M. a cura di 1997, Le Terramare. La più antica civiltà padana, Milano, Electa, 107-125.

CREMASCHI M., 2009a. Foreste, terre coltivate, e acque, l'originalità del progetto terramaricolo. In: M.A. Bernabò Brea, M Cremaschi a cura di, Acque e Civiltà nelle terramare. La Vasca votiva di Noceto. Università degli Studi di Milano, Le finestre del Sapere 7, Skira, Milano, 34-45.

CREMASCHI M., 2009b. Ambiente, clima ed uso del suolo nella crisi della cultura delle terramare. In Cazzella A., Cardarelli A., Frangipane M., Peroni R. (a cura di), Atti del Convegno Le Ragioni del Cambiamento, Atti del Convegno Internazionale (Roma 2006), Scienze dell'Antichità, 15. 521-534.

Cremaschi M., Pizzi C., Valsecchi V., 2006. Water management and land use in the terramare and a possible climathic co-factor in their collapse. The case study of the terramara S. Rosa (Northern Italy), in Quaternary International, 151. 87-98.

Cremaschi M., Pizzi C., 2007. I pozzi perimetrali del Villaggio Grande di Santa Rosa di Poviglio (RE). Uso delle risorse idriche tra la fine del Bronzo Medio e il Bronzo Recente, in Studi in onore di Renato Peroni, Roma, 117-128.

Cremaschi M., Nicosia C., 2012. Sub-Boreal aggradation along the Apennines margin of the central Po plain: geomorphological and geoarchaeological aspects. Geomorphologie, 2, 156-174.

Cremaschi M., Pizzi C., 2013. La recinzione e il terrapieno al margine del Villaggio Grande della Terramara Santa Rosa di Poviglio, in Atti del Convegno Mura di legno, mura di terra, mura di pietra: fortificazioni nel Mediterraneo antico (Roma, 7-9 maggio 2012). Scienze dell'Antichità, 19. 25-26.

Cremaschi M., Mercuri A.M., Torri P., Florenzano A., Pizzi C., Marchesini M., ZERBOnI A., 2016 a. Age: new insights from the VP/VG sequence of the Terramara Santa Rosa di Poviglio. Quaternary Science Reviews, 136. 153-172.

Cremaschi M., Mutti A., Baratti G., Borgi F., Brandolini F., Donati N., Ferrari P., Fronza G., Lachenal T., Zerboni A., 2016 b. La terramara Santa Rosa di Poviglio: strutture tra Villaggio Piccolo e Villaggio Grande. Nuovi dati dallo scavo 2015. The Journal of FASTI ON LINE 349.

De Grossi Mazzorin J., Riedel A., 1997. La fauna delle terramare, Bernabò Brea M., Cardarelli A., Cremaschi M. a cura di, 1997, Le Terramare. La più antica civiltà padana, Milano, Electa, 475-480.

De Marinis R., 1975. L'età del Bronzo, in Cremaschi M., a cura di, Preistoria e Protostoria nel Reggiano, Reggio Emilia, Civici Musei, pp. 31-53.

FORNI G., 1997. Le tecniche agricole nelle terramare, in Bernabò Brea M., Cardarelli A., Cremaschi M. a cura di, 1997, Le Terramare. La più antica civiltà padana, Milano, Electa.457-464.

Holzhauser H., Magny M., Zumbuhl H.J., 2005. Glacier and lake-level variations in western-central Europe over the last 3500 years, The Holocene 15. 789-801.

LANCELOTTI C., 2004-2005. Archeobotanica ed aspetti archeologici delle unità US 3 e US 234 della Palafitta di Parma, Università degli Studi di Milano, Master "Scienze per i Beni Culturali Archeologici: Figure professionali di geoarcheologo ed archeometra", Anno Accademico 2004-2005. 
Le Roy Ladurie E., 2004. Histoire humaine et comparée du climat. I. Canicules et Glaciers XIII-XVIII siècles, Paris, Fayard.

Magny M., 2004. Holocene climate variability as reflected by mid-European lake-level fluctuations and its probable impact on prehistoric buman settlements, in Brauer A., Guilizzoni P., a cura di, The record of Human/ Climate Interaction in Lake Sediments, Quaternary International 113, 65-79.

Mele M., Cremaschi M., Giudici M., Lozej A., Pizzi C., Bassi A., 2013. The terramare and the surrounding bydraulic structures: a geophysical survey of the Santa Rosa site at Poviglio (Bronze age, Northern Italy, Journal of Archaeological Science, 40, 4648-4662.

Mercuri A.M., Accorsi C.A., Bandini Mazzanti M., Bosi G., Trevisan Grandi G., Cardarelli A., Labate D., Olmi L., Torri P., 2006. Cereal Fields from the Middle-Recent Bronze Age as found in the terramara di Montale, in the Po plain (Emilia Romagna, Northern Italy), based on pollen, seeds/fruits and microcharcoals, in Morel J.-P., Tresserras Juan J., Matamala J.C., a cura di, The Archaeology of Crop fields and garden Archaeology, Bari, Edipuglia, 251-270.

Mercuri A.M., Accorsi C.A., Bandini Mazzanti M., Bosi G., Cardarelli A., Labate D., Marchesini M., Trevisan Grandi G., 2006. Economy and environment of Bronze age settlemnets - Terramaras - on the Po plain (Northern Italy): first results from the archeobotanical research at the Terramara di Montale, in Vegetation History and Archaeobotany 16, 43-60.

Orombelli G., 1997. Le condizioni climatiche durante il II millennio a.C., in Bernabò Brea M., Cardarelli A., Cremaschi M. a cura di 1997, Le Terramare. La più antica civiltà padana, Milano, Electa. 51-58.

PEARCE M., 1998. New research on the terramare of northern Italy, Antiquity 72, pp. 743-746.

Pizzi C., Cremaschi M., 2004. I pozzi lungo la struttura di recinzione del Villaggio Grande di Poviglio: caratteri e processi formativi, in Cocchi Genick D., a cura di, L'età del Bronzo recente in Italia, Atti del Congresso, Viareggio, Mauro Baroni. 512-513.

Ravazzi C., Cremaschi M., Forlani L., 2004. Studio archeopalinologico. Nuovi dati, analisi floristica e sintassonomica della vegetazione nell' età del Bronzo, in Bernabò Brea M., Cremaschi M., a cura di, 2004b, Il Villaggio Piccolo della Terramara Santa Rosa a Poviglio. Scavi 1987-1992, Origines, Firenze, pp. 703-736.

Regattieri E., Zanchetta G., Drysdale R.N., Isola I., Hellstrom J.C., Dallai L., 2014. Lateglacial to Holocene trace element record ( $B a, M g$, Sr) from Corchia Cave (Apuan Alps, central Italy): paleoenvironmental implications. Journal of Quaternary Science, 29, 4, 381-392.

Riedel A., 2004. La fauna. In: Bernabò Brea M., Cremaschi M., a cura di, 2004, Il Villaggio Piccolo della Terramara Santa Rosa a Poviglio. Scavi 1987-1992, Origines, Firenze, 703-736.

Valsecchi V., Finsinger W., Tinner W., Amman B., 2008. Testing the influence of climate, buman impact, and fire on the Holocene population expansion of Fagus sylvatica in the southern Prealps (Italy), The Holocene 18, 4, 603-614. 